Case Report

\title{
Central Venous Line Insertion Revealing Partial Anomalous Pulmonary Venous Return: Diagnosis and Management
}

\author{
Bashar Alzghoul, ${ }^{1}$ Ayoub Innabi, ${ }^{1}$ Aditya Chada, ${ }^{2}$ Ahmad R. Tarawneh, ${ }^{2}$ \\ Krishna Kakkera, ${ }^{2}$ and Khaled Khasawneh ${ }^{2}$ \\ ${ }^{1}$ Department of Internal Medicine, University of Arkansas for Medical Sciences, Little Rock, AR, USA \\ ${ }^{2}$ Division of Pulmonary and Critical Care Medicine, University of Arkansas for Medical Sciences, Little Rock, AR, USA \\ Correspondence should be addressed to Bashar Alzghoul; bnalzghoul@uams.edu
}

Received 5 April 2017; Accepted 9 May 2017; Published 29 May 2017

Academic Editor: Gil Klinger

Copyright (C) 2017 Bashar Alzghoul et al. This is an open access article distributed under the Creative Commons Attribution License, which permits unrestricted use, distribution, and reproduction in any medium, provided the original work is properly cited.

Central venous line malposition is a well-known complication of line insertion. Rarely, it can be mal-positioned in an anomalous pulmonary vein. We present an unusual case of a 56-year-old woman that was found to have partial anomalous pulmonary venous return on central venous line insertion. In this report, we describe a systematic approach to diagnosis and management of this unusual situation.

\section{Introduction}

Partial anomalous pulmonary venous return (PAPVR) is a rare congenital anomaly that occurs during embryologic development when at least one pulmonary vein fails to communicate with the left atrium; instead, it drains directly or indirectly in the right atrium. It remains asymptomatic most of the time and is discovered incidentally or postmortem [1]. There have been few reports in literature of PAPVR discovered after central venous line (CVL) insertion. We report a 56-year-old woman that was found to have left upper PAPVR on CVL insertion. In addition, we conducted literature review of similar cases reported before and suggested a systematic approach to diagnosis and management.

\section{Case Presentation}

A 56-year-old woman with a medical history of multiple sclerosis, quadriplegia, sacral decubitus ulcer, chronic osteomyelitis, chronic deep vein thrombosis (DVT), chronic steroids use, and chronic Foley catheter use presented to the emergency department with severe sepsis due to multiple potential sources including urinary tract infection, soft tissue infection, or osteomyelitis. Vital signs were significant for blood pressure of $85 / 59 \mathrm{mmHg}$, heart rate of 63 beats per minute, respiratory rate of 33 breaths per minute, and oxygen saturation of $83 \%$ on room air. At the beginning, patient declined intubation so she was placed on $100 \%$ oxygen via nonrebreather mask. Arterial blood gas (ABG) on 100\% oxygen was 7.29/49/55. Initial chest X-ray (CXR) showed small bilateral pleural effusions with suspected left lower lobe infiltrate. Despite intravenous (IV) fluid resuscitation, broad spectrum IV antibiotics, and stress dose steroids, patient became more hypotensive and IV norepinephrine was started through a right femoral CVL that was placed in the emergency department. Patient was started on heparin drip for suspected pulmonary embolism that was stopped later due to large bleeding from femoral line site and consideration of pneumonia as the cause of hypoxemia. Patient agreed later to be intubated and decision was made to remove femoral line and place a 7.0 Fr triple-lumen CVL through the left internal jugular vein (IJ) over a guidewire and under full ultrasound (U/S) guidance, which was placed easily with no complications. A CXR was obtained to confirm placement which showed that the catheter tip did not cross the midline; instead, it was extending towards the left of the aortic arch probably into an anomalous vein as read by the radiologists (Figure 1). Ultrasound examination of the neck showed 


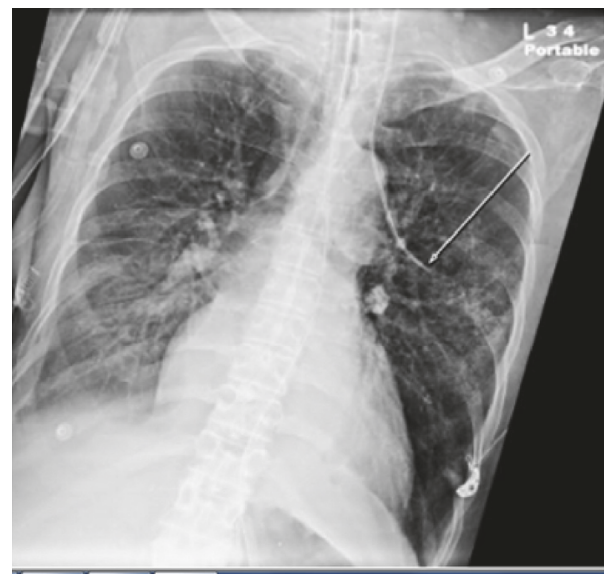

Figure 1: Single frontal view CXR showing the tip of the left IJ CVL extending left of the aortic arch towards the left lung field.

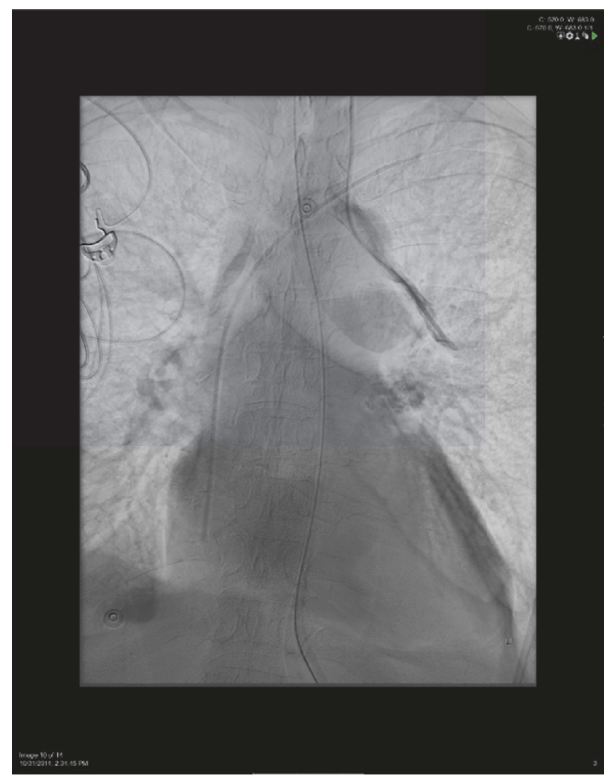

FIGURE 2: Fluoroscopy study showing left IJ CVL extending through the anomalous pulmonary vein.

proper catheter placement in the left IJ. A pressure transducer was connected which showed a venous wave form with a pressure of $10 \mathrm{mmHg}$. ABG obtained from the catheter on continuous mechanical ventilation $\left(\mathrm{FiO}_{2}=50 \%\right.$, positive end expiratory pressure $=5 \mathrm{CM} \mathrm{H} \mathrm{H}_{2} \mathrm{O}$ ) showed $\mathrm{PaO}_{2}$ of $102 \mathrm{mmHg}$ compared to $79 \mathrm{mmHg}$ from radial arterial site. A transthoracic echocardiography, which was done to evaluate cardiac function, did not show evidence of atrial septal defect or any other cardiac malformation. Fluoroscopy study was used to place a left peripherally inserted central catheter (PICC) and to confirm the location of the left IJ CVL which demonstrated contrast injection in an anomalous pulmonary vein (Figure 2). We concluded that the patient has partial anomalous pulmonary venous return (PAPVR) and the left IJ CVL was removed. The PICC line was used instead for central access.

\section{Discussion}

Central venous lines (CVLs) insertion is a very common procedure that is performed by practitioners of different specialties for varied indications. Among these indications are drug administration, parenteral nutrition, renal replacement therapy, cardiac catheterization, and trans-venous pacing of the heart [2]. Malposition of CVL insertion is relatively common due to operator and technical factors in part. However, it can be due to congenital and acquired abnormalities of the venous system that leads to abnormal positioning of the line. In some cases, CVL malposition can lead to catastrophic complications $[3,4]$. Thus, it is critically important for practitioners to identify malposition of CVLs and take evidence based appropriate actions depending on the situation.

CXR is routinely used to confirm the location of the CVL, which ideally should be at the junction between the 


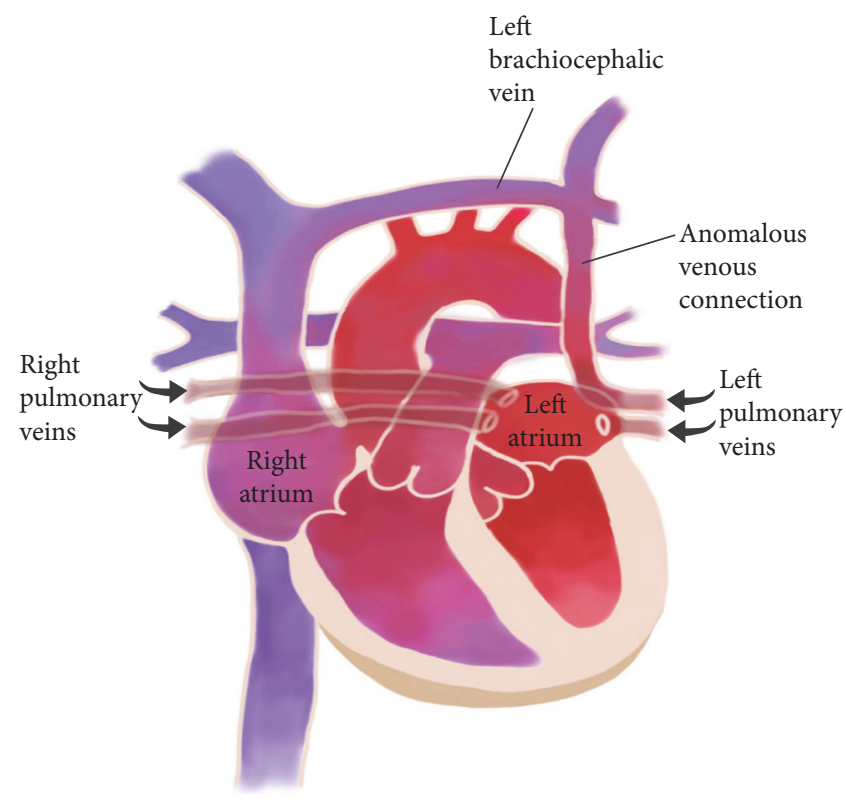

FIGURE 3: Schematic illustration of the anatomy of the CVL inserted accidentally in an anomalous pulmonary vein connected to the right atrium via the brachiocephalic vein.

superior vena cava (SVC) and the right atrium. There are multiple radiographic landmarks that can be utilized on CXR to confirm that the CVL is in the right location [5]. In our case, we encountered an unusual location of the CVL on the CXR where the catheter tip did not cross the midline; instead, it was extending laterally towards the left lung field, left of the aortic arch. The CVL was clearly mal-positioned, but the question that we had was where exactly was the line inserted: Was it inserted in the carotid artery? Does the patient have a persistent left SVC?

We decided to follow a systematic approach that showed the CVL was inserted in an anomalous pulmonary vein. This finding might be encountered by other practitioners in critical situations where someone needs to make fast decisions, so it is important to be familiar with the anatomy.

The pulmonary veins originally arise from a common pulmonary vein that buds from the left atrium during the embryologic development. It then joins the splanchnic venous plexus in the lung buds. If any or all of the pulmonary veins fail to connect to the left atrium, it will eventually divert the blood directly or indirectly to the right atrium $[1,6]$. Figure 3 shows a schematic view of left upper partial anomalous pulmonary venous return (PAPVR). On cadaveric studies, PAPVR was reported in up to $0.7 \%$ of the cases [1]. It was first described by Winslow in 1739 [7, 8]. There are scarce cases in literature of accidental diagnosis of PAPVR in adults after CVL insertion. We did a literature review using PubMed and Google scholar search engines using the key words (Central Venous) and (partial anomalous). We included cases of adult patients who were diagnosed to have PAPVR after CVL insertion and ended up with 10 cases. As illustrated in Table 1, we managed to summarize the approach that was followed in these cases. We tried in this article to highlight the diagnostic and management approach followed in other reported cases and suggest a systematic approach to follow when encountering similar situation.

First we used the U/S to examine the course of the CVL in the neck to confirm whether the CVL was inserted in the IJ vein or in the carotid artery. Of note, U/S examination of the chest can be used sometimes to localize the tip of CVL; however, it needs an experienced operator and may not detect the final intrathoracic placement of the CVL all the time [9]. Second, we connected the CVL to a pressure transducer which showed a venous wave form. Among the cases reported in literature, Wylam and Schmidt [6] were the only to describe pulsating arterial wave form. They attributed that to wedging of the tip of the catheter in a pulmonary venous branch, which would subsequently reflect the pressure tracing from that of the upstream pulmonary artery. However, in their case, the patient had moderate pulmonary hypertension diagnosed by heart catheterization. Third, we obtained ABG from the CVL and from arterial site. In our case, the $\mathrm{PaO} 2$ was paradoxically higher from the CVL compared to arterial sample. This paradoxical finding was reported in four of the 10 cases $[6,10-12]$. In addition, $\mathrm{PO}_{2}$ was reported to be high in four other cases, but no arterial sample was obtained to compare [13-16]. The high $\mathrm{PO}_{2}$ can be explained by the fact that the catheter tip is collecting blood directly from the pulmonary venous blood flow before intermixing with systemic venous blood [15]. The fourth step would be to confirm placement via definitive radiologic method. In our case, since we decided to substitute the CVL with a PICC, we used fluoroscopy studies to define the anatomy. From this, it might be unnecessary to use CT/CTA only to establish the diagnosis of CVL insertion in an aberrant pulmonary vein unless it was indicated for some other reason. 


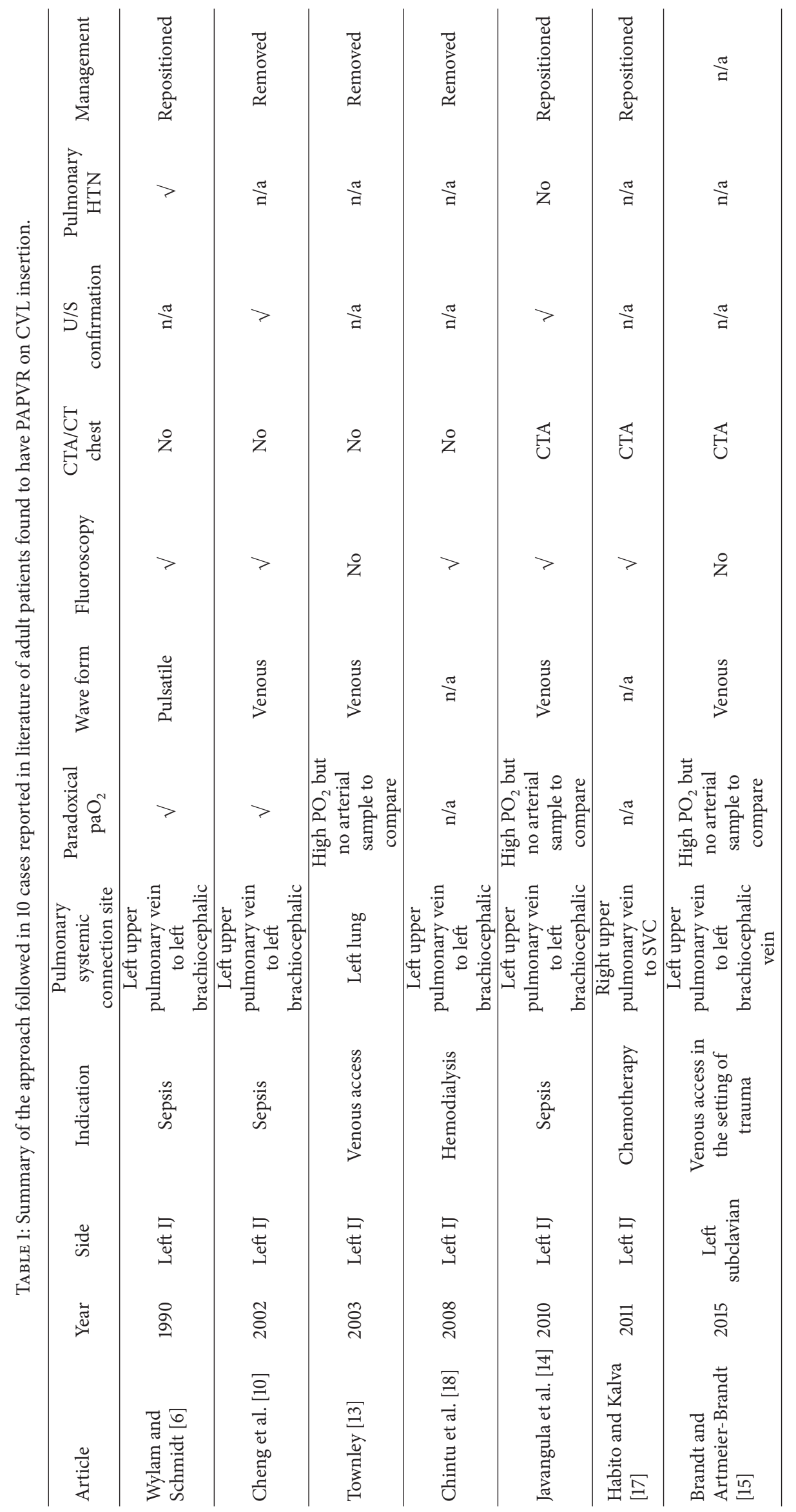




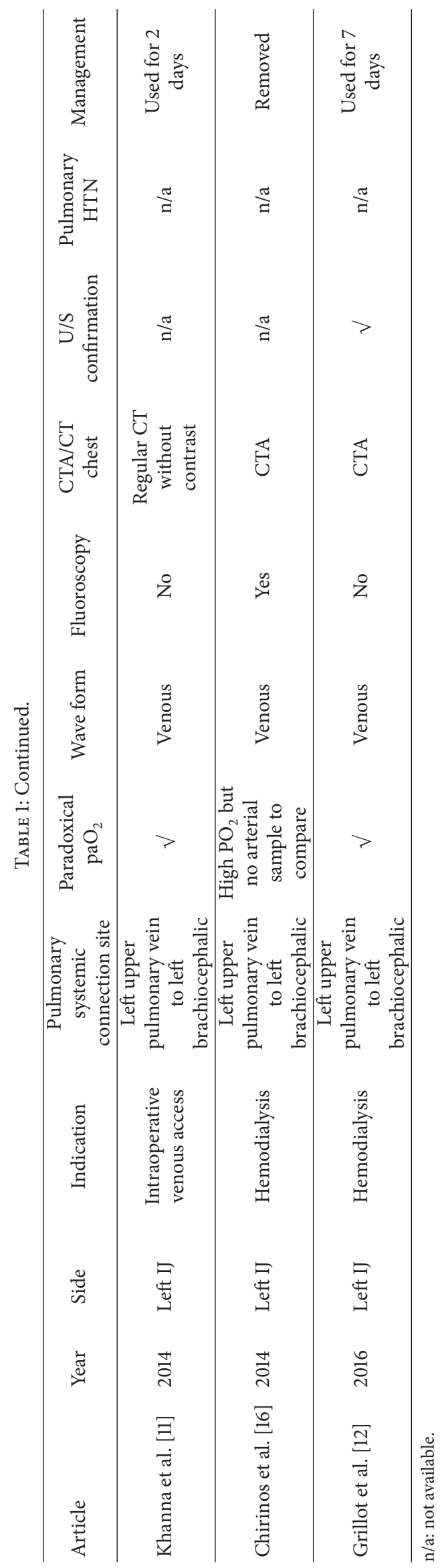


Instead, one can use the constellation of findings described above and confirm the diagnosis using simple fluoroscopy studies.

There are no enough cases in literature to examine the safety of using the CVL inserted in an anomalous pulmonary vein. In our case, we opted to remove the catheter since we had alternative options. Other authors opted for reposition of the catheter under fluoroscopy guidance $[6,14,17]$. However, Khanna and colleagues [11] reported using the CVL safely for two days as venous access. In addition, Grillot and colleagues [12] reported using it for seven days for hemodialysis.

\section{Conclusion}

It is important to be familiar with PAPVR as a cause of CVL malposition. When encountered, the systematic approach can help establish the diagnosis. This includes ultrasound confirmation of venous access, checking blood gases from the CVL and concurrently from an arterial site which is expected to show high $\mathrm{PO}_{2}$ that might be paradoxically higher than the arterial side, checking the pressure wave, and using fluoroscopy to confirm radiologically.

\section{Conflicts of Interest}

The authors declare that there are no conflicts of interest regarding the publication of this article.

\section{Acknowledgments}

The authors express great gratitude for Maya Amer, BAR, for graphic design and assistance.

\section{References}

[1] J. E. J. Healy, "An anatomic survey of anomalous pulmonary veins: their clinical significance," The Journal of Thoracic Surgery, vol. 23, pp. 433-444, 1952.

[2] P. L. Tan and M. Gibson, "Central venous catheters: the role of radiology," Clin. Radiol, vol. 61, pp. 13-22, 2006.

[3] F. Gibson and A. Bodenham, "Misplaced central venous catheters: applied anatomy and practical management," British Journal of Anesthesia, vol. 110, 2013.

[4] L. Wang, Z.-S. Liu, and C.-A. Wang, "Malposition of central venous catheter: presentation and management," Chinese Medical Journal, vol. 129, no. 2, pp. 227-234, 2016.

[5] M. Wirsing, C. Schummer, R. Neumann, J. Steenbeck, P. Schmidt, and W. Schummer, "Is traditional reading of the bedside chest radiograph appropriate to detect intraatrial central venous catheter position?" Chest, vol. 134, no. 3, pp. 527-533, 2008.

[6] M. E. Wylam and G. A. Schmidt, "Serendipitous discovery during jugular catheterization. Partial anomalous pulmonary venous connection," Chest, vol. 98, no. 2, pp. 493-495, 1990.

[7] Winslow. No Title. J. Mem Acad Roy Sci. 113 (1739).

[8] N. M. Ammash, J. B. Seward, C. A. Warnes, H. M. Connolly, P. W. O'Leary, and G. K. Danielson, "Partial anomalous pulmonary venous connection: Diagnosis by transesophageal echocardiography," Journal of the American College of Cardiology, vol. 29, no. 6, pp. 1351-1358, 1997.
[9] H. Kamalipour, S. Ahmadi, K. Kamali, A. Moaref, M. Shafa, and P. Kamalipour, "Ultrasound for localization of central venous catheter: a good alternative to chest X-ray?" Anesthesiology and Pain Medicine, vol. 6, no. 5, Article ID e38834, 2016.

[10] C. J. C. Cheng, B. L. Lim, and B. S. Tan, "Inadvertent catheterisation of a partial anomalous pulmonary venous channel during central venous cannulation," Anaesthesia, vol. 57, no. 2, pp. 198200, 2002.

[11] A. K. Khanna, A. Maheshwari, M. J. Popovich, and P. Mathur, "Incidental discovery of a partial anomalous pulmonary venous connection in the surgical critical care unit," Journal of Clinical Anesthesia, vol. 26, 2014.

[12] N. Grillot, S. Figueiredo, A. Aubry, P.-E. Leblanc, and J. Duranteau, "Unusual dialysis catheter position due to partial anomalous pulmonary venous return: diagnosis and management," Anaesthesia Critical Care and Pain Medicine, vol. 35, no. 3, pp. 233-235, 2016.

[13] S. A. Townley, "Central venous catheter malposition in an anomalous pulmonary vein," European Journal of Anaesthesiology, vol. 20, no. 12, pp. 985-986, 2003.

[14] K. Javangula, J. Cole, M. Cross, and P. H. Kay, "An unusual manifestation of left partial anomalous pulmonary venous connection," Interactive Cardiovascular and Thoracic Surgery, vol. 11, no. 6, pp. 846-847, 2010.

[15] L. Brandt and U. Artmeier-Brandt, "Partial anomalous pulmonary venous connection as incidental finding: explanation for apparently paradoxical central venous blood gas analysis results," Anaesthesist, vol. 64, no. 10, pp. 754-757, 2015.

[16] J. C. Chirinos, J. A. Neyra, J. Patel, and A. R. Rodan, "Hemodialysis catheter insertion: is increased $\mathrm{PO}_{2}$ a sign of arterial cannulation? A case report," BMC Nephrology, vol. 15, article 127, 2014.

[17] C. M. R. Habito and S. P. Kalva, "Partial Anomalous Pulmonary Venous Return Detected Incidentally during Port Placement," J. Vasc. Interv. Radiol, vol. 23, 2012.

[18] M. R. Chintu, S. Chinnappa, and S. Bhandari, "Aberrant positioning of a central venous dialysis catheter to reveal a left-sided partial anomalous pulmonary venous connection," Vascular Health and Risk Management, vol. 4, no. 5, pp. 1141-1143, 2008. 


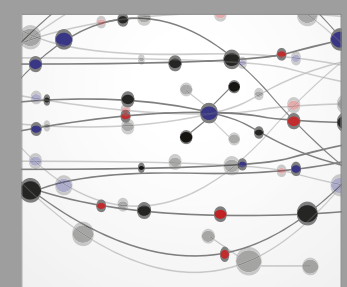

The Scientific World Journal
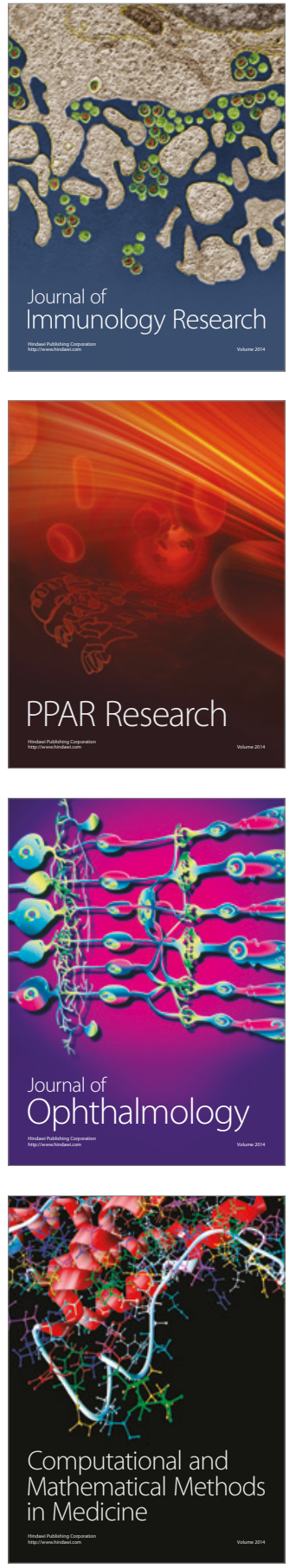

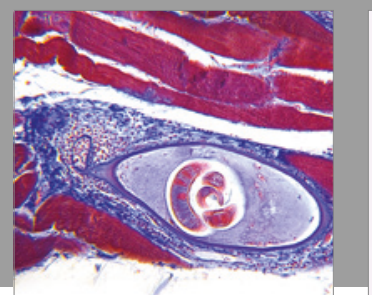

Gastroenterology Research and Practice
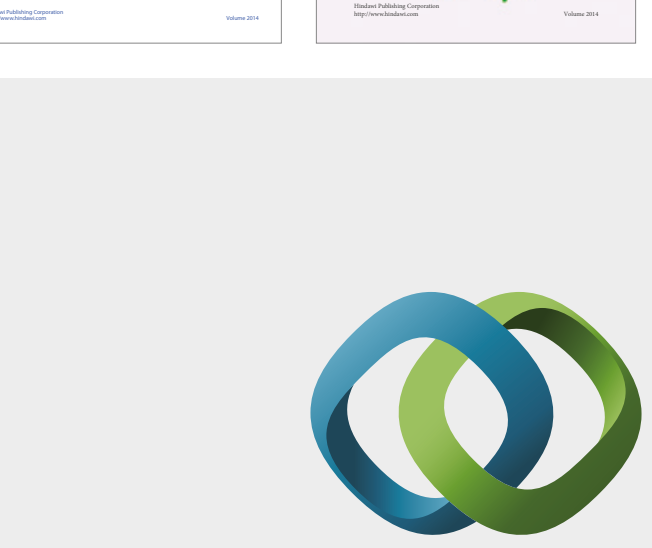

\section{Hindawi}

Submit your manuscripts at

https://www.hindawi.com
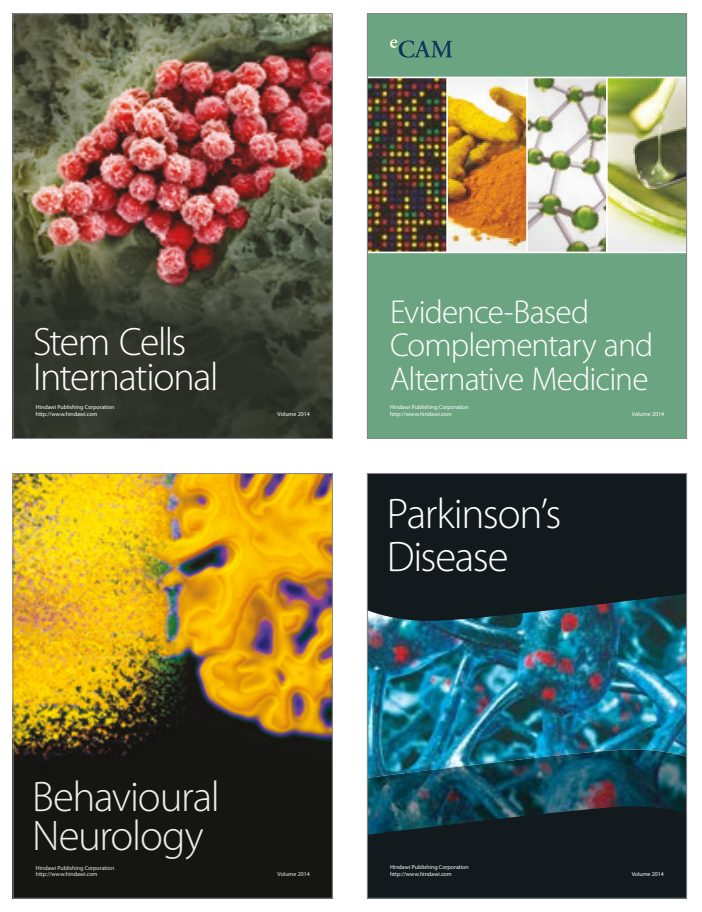
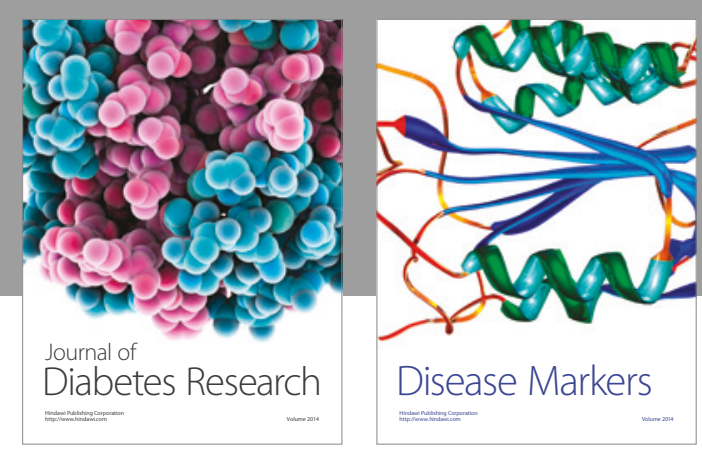

Disease Markers
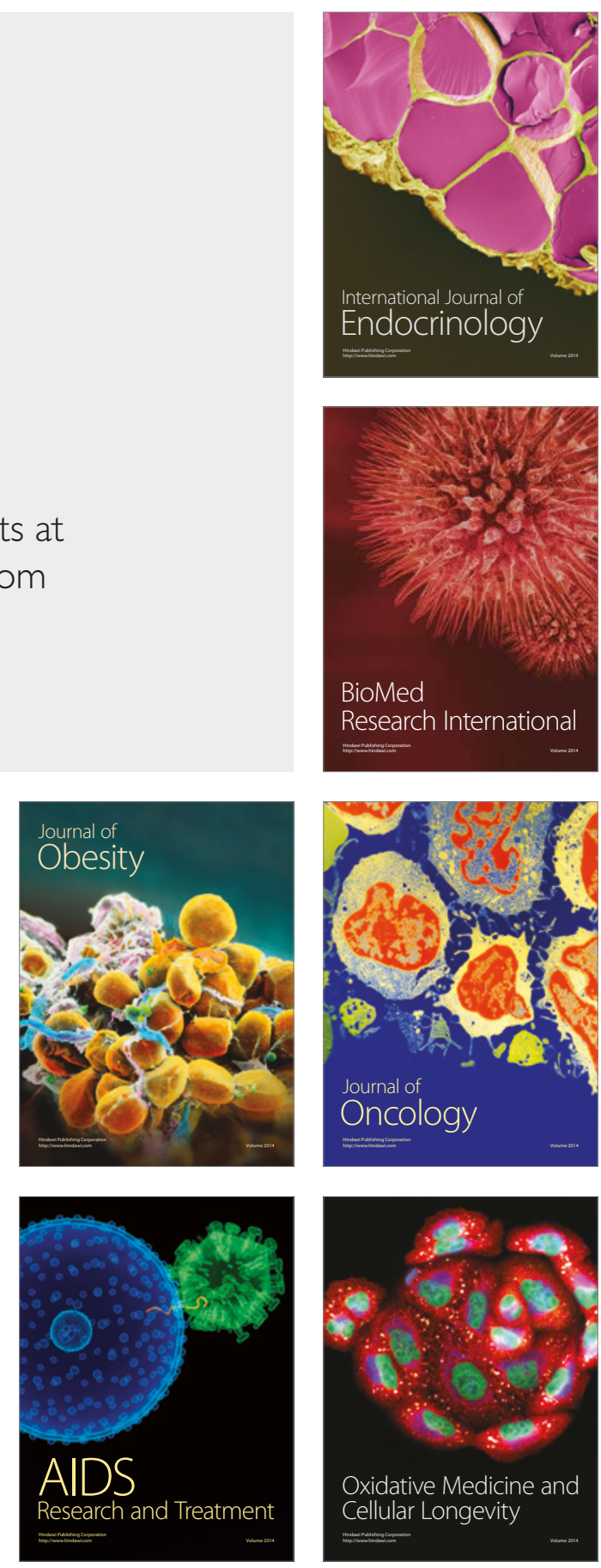